\title{
Application of Growth Functions to Describe the Dynamics of Avascular Tumor in Human Body
}

\author{
Bayru Haftu Hindeya, Samba Narasimha Murthy* \\ School of Mathematical and Statistical Sciences, Hawassa University, Hawassa, Ethiopia \\ Email address: \\ bayru10h@gmail.com (B. H. Hindeya), simhamurthy44@gmail.com (S. N. Murthy) \\ ${ }^{*}$ Corresponding author
}

To cite this article:

Bayru Haftu Hindeya, Samba Narasimha Murthy. Application of Growth Functions to Describe the Dynamics of Avascular Tumor in Human Body. Applied and Computational Mathematics. Vol. 5, No. 2, 2016, pp. 83-90. doi: 10.11648/j.acm.20160502.18

Received: April 20, 2016; Accepted: April 29, 2016; Published: May 12, 2016

\begin{abstract}
This paper deals with the applications of mathematical growth functions such as monomolecular, time delay logistic and Gompertz functions to describe the dynamics of avascular tumor growth. In this case we analyze the steady state of the modified systems of the model using Jacobean matrix to show that it is stable on the nontrivial stationary points of each applications. Numerical simulation of the growth functions is implemented by using "ode45" in MATLAB and graphical outputs are presented to show differences in evaluation of tumor sub-populations. We also find that the tumor cells increases with time so that the nutrient is disproportional to the number of cells and they transform in to quiescent and necrotic cells that cause cancer.
\end{abstract}

Keywords: Avascular, Tumor, Monomolecular, Time Delay Logistic, Gompertz, Proliferating Cells, Quiescent Cells, Necrotic Cells

\section{Introduction}

The development of a solid tumor begins with a single cell that can be transformed as a result of mutation. Unlike the normal cell the transformed cell scope homeostatic mechanisms of the body with inappropriate proliferation and leads to override apoptosis. Every tumor cell divides freely to develop in to a cluster of tumor cells to form a massive tissue termed as spheroid. Further, growth and proliferation of the tumor leads to the development of an avascular tumors consisting of around $10^{6}$ cells which feed only on the nutrients available in the local environment [12]. The avascular spheroids structurally consists of the outer most zone dominantly composed of proliferating cells, the inner most zone of the necrotic or dead cells and the middle layer largely composed of quiescent cells. As a starting point to minimize complexity of all stages of cancer, most literates agree that avascular tumor growth study is a basic foundation of the problem.

Mathematical modeling and analysis of tumor growth processes give important insights on cancer growth situations. It is commonly believed that tumor growth under ideal conditions is a simple exponential process assumed that sufficient nutritional support is provided by the host. However, a survey of literature shows that exponential growth of tumors has been observed only rarely and for relatively brief periods mainly at its very early stage. When we consider tumors whose growth has been followed over time, they grow more and more slowly as their spheroid get larger [1]. Recently mathematical models and numerical simulation with different approaches have been developed to describe features of avascular tumor growth. For simplicity of mass balance clothing system, tumor cells are assumed to be identical [12, 15 - 17].

Therefore, this work assess the application of monomolecular, time delay logistic, and Gompertz growth functions and their numerical analysis for dynamics of avascular tumor growth model. Indeed numerical simulation of growth functions will be implemented by using MATLAB.

\section{Growth Model of Avascular Tumor}

\subsection{Heterogeneous Model for Dynamics of Avascular Tumor Growth}

The increment in size of neoplasm, new and abnormal growth of tissue, caused by abnormaldivision among 
malignant cells is one of the most feasible observations under the investigation of cancer. Such studies may answer as to how the size of a tumor increases through time.

In the present work we find a modified heterogeneous model on dynamics of avascular tumor growth as combinations of models stated in $[4,5]$ on one hand and on the other hand are considered with the application of growth functions. We construct three compartmental models of growth functions that specifically predict time delay logistic growth, Gompertz growth and monomolecular growth functions for the number of cells $\mathrm{N}(\mathrm{t})$ in the tumor. Accordingly the following model is a general assumption for the application of growth functions.

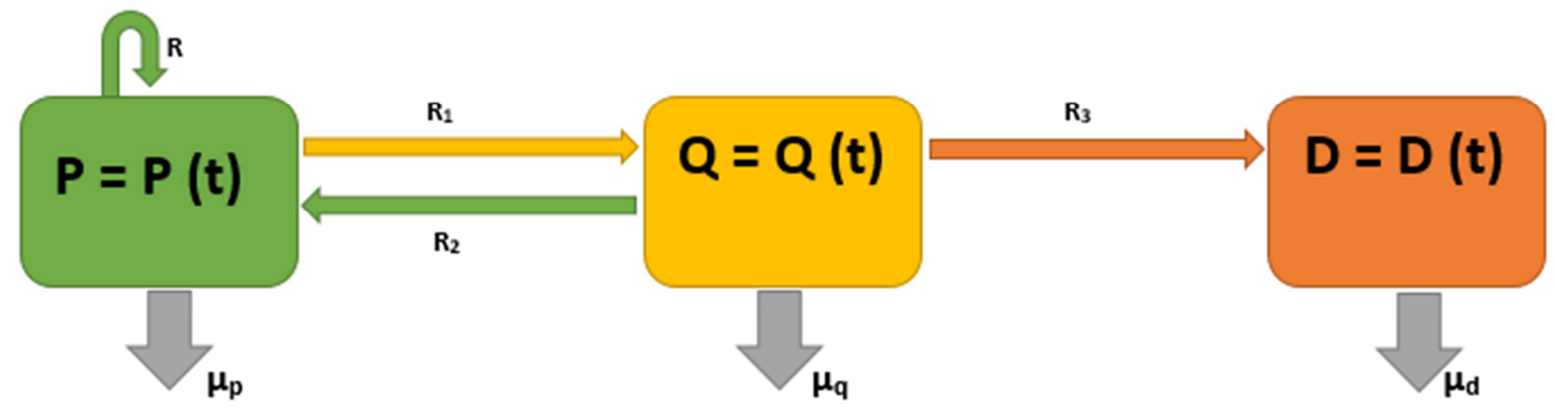

Figure 1. Schematic diagram for growth function of tumor cells.

$$
\begin{aligned}
& \frac{d P}{\mathrm{dt}}=\left[\mathrm{R}-\mathrm{R}_{1}-\mu_{\mathrm{p}}\right] \mathrm{P}+\mathrm{R}_{2} \mathrm{Q} \\
& \frac{d Q}{\mathrm{dt}}=\mathrm{R}_{1} \mathrm{P}-\left(\mathrm{R}_{2}+\mathrm{R}_{3}+\mu_{\mathrm{q}}\right) \mathrm{Q} \\
& \frac{d D}{\mathrm{dt}}=\mathrm{R}_{3} \mathrm{Q}-\mu_{\mathrm{d}} \mathrm{D}
\end{aligned}
$$

Here in the system (1), $\mathrm{N}=\mathrm{P}+\mathrm{Q}+\mathrm{D}, \mathrm{N}_{0}=\mathrm{P}_{0}+\mathrm{Q}_{0}+\mathrm{D}_{0}=1$. Also the rate parameters are positive; $\mathrm{P}, \mathrm{Q}$, Dare proliferating, quiescent, dead or necrotic cells respectively, Ris proliferating rate or mitosis of cells, $\mu_{\mathrm{p}}$ is natural exiting rate of proliferating cells or apoptosis, $\mu_{\mathrm{q}}$ is natural exiting rate of quiescent cells apoptosis, $\mu_{\mathrm{d}}$ is natural exiting rate of necrotic cells or apoptosis, $\mathrm{R}_{1}$ is transition rate from proliferating to quiescent cells, $R_{2}$ is transition rate from quiescent to proliferating cells and $R_{3}$ is transition rate from quiescent to dead or necrotic cells.

\subsection{Introduction to Basic Growth Functions}

Qualitatively, the growth of an animal can be divided into four stages such that early exponential growth, where the rate of growth is proportional to weight; linear growth, where more and more energy is devoted to maintenance; diminishing growth as a maintenance balance is approached; and antithesis through senescence which often disregarded since few or no observations are made [3]. It is commonly believed that ideally tumor growth is a simple exponential process. Exponential growth describes the ideal growth status in which cells population divide without constraint, and continue to double indefinitely. However, limitations of resources like availability of nutrients, oxygen and space imply that exponential growth is impractical for the long term growth of solid tumors. Hence this model is effective for early tumor growth stage called avascular and rate of population growth declines as population size increases.

To overcome the problem of simple exponential growth and to fit with real world manifestation of cells growth so as to consider its saturation, Koya-Goshu [9] generalized biological growth is one way from which multiple growth functions such as Logistic, Generalized Logistic, logistic delay, Gompertz, Brody, Monomolecular, Mitscherlich, Von Bertalanffy, Richards, Generalized Weibull and Weibull functions can be derived.

Koya-Goshu generalized growth function at integral constant $C=1-\left(\frac{K_{t^{*}-K_{0}}}{\mathrm{~K}-K_{0}}\right)^{\frac{1}{\mathrm{~m}}}$ is defined as

$$
f(t)=K_{0}+\left(\mathrm{K}-K_{0}\right)\left(1-\mathrm{C} \boldsymbol{e}^{-K\left(\frac{t-t^{*}}{\delta}\right)^{v}}\right)^{m}
$$

Among all derivations of Koya-Goshu generalized biological growth model, this work is centered at the monomolecular, time delay logistic and Gompertz growth functions. These models together with their multiple response and multivariate generalizations are now widely used in applied research for modeling and forecasting behavior of population growth dynamics. Inequation (2) the parameters used are interpreted as shown in Table 1.

Table 1. Parameters description for Koya-Goshu growth function.

\begin{tabular}{ll}
\hline Parameters & Description of parameters \\
\hline K: $\lim _{t \rightarrow \infty} f(t)=f$ & Upper asymptote (carrying capacity) of $f(t)$ \\
$K_{0}$ & Lower asymptote of $f(t)$ \\
$K_{t^{*}}=f\left(t^{*}\right)$ & Growth rate parameter of $f(t)$ \\
$t^{*}$ & A constant time shift of $f(t)$ \\
$\delta$ & A constant time scale of $f(t)$ \\
$v, m: v m \neq 0$ & Shape parameters of the growth function \\
\hline
\end{tabular}

\section{Application of Basic Growth Functions}

Monomolecular, logistic time delay and Gompertz functions are applicable on the model for dynamics of avascular tumor growth. Basically cell densities of 
proliferating, quiescent and necrotic cells are available in the outer, middle and inner zones of the tumor respectively which feed on the common nutrients around it. Applications of the growth functions are concentrated on the proliferating cells which are capable to reproduce and grow number of cells in the spheroid.

The relative growth rate, growth function and their corresponding ODE of the three growth functions known as monomolecular, delay logistic and Gompertz is summarized in the following Table 2.

Table 2. Summary on Mathematical expressions of growth functions.

\begin{tabular}{llll}
\hline Model's Name & Relative growth rate & Growth function & Corresponding ODE \\
\hline Monomolecular & $r_{\mathrm{t}}=r\left(\frac{K}{f(t)}-1\right)$ & $f(t)=K\left(1-\mathrm{C} e^{-r t}\right)$ & $\frac{d f(t)}{d t}=r(K-f(t))$ \\
Delay Logistic & $r_{\mathrm{t}}=r\left(1-\frac{f(t-\tau)}{K}\right)$ & $f(t)=\frac{K}{1+C e^{-r t}}$ & $\frac{d f(t)}{d t}=r f(t)\left(1-\frac{f(t-\tau)}{K}\right)$ \\
Gompertz & $r_{\mathrm{t}}=r \log \frac{K}{f(t)}$ & $f(t)=K e^{-\exp (-r t)}$ & $\frac{d f(t)}{d t}=r f(t) \log \frac{K}{f(t)}$ \\
\hline
\end{tabular}

The nature of monomolecular, time delay logistic and Gompertz functions generate sigmoid curves as exemplified in figure 2 which indicate the saturation of population growth applied for the growth of living thing such as cells inside the body.

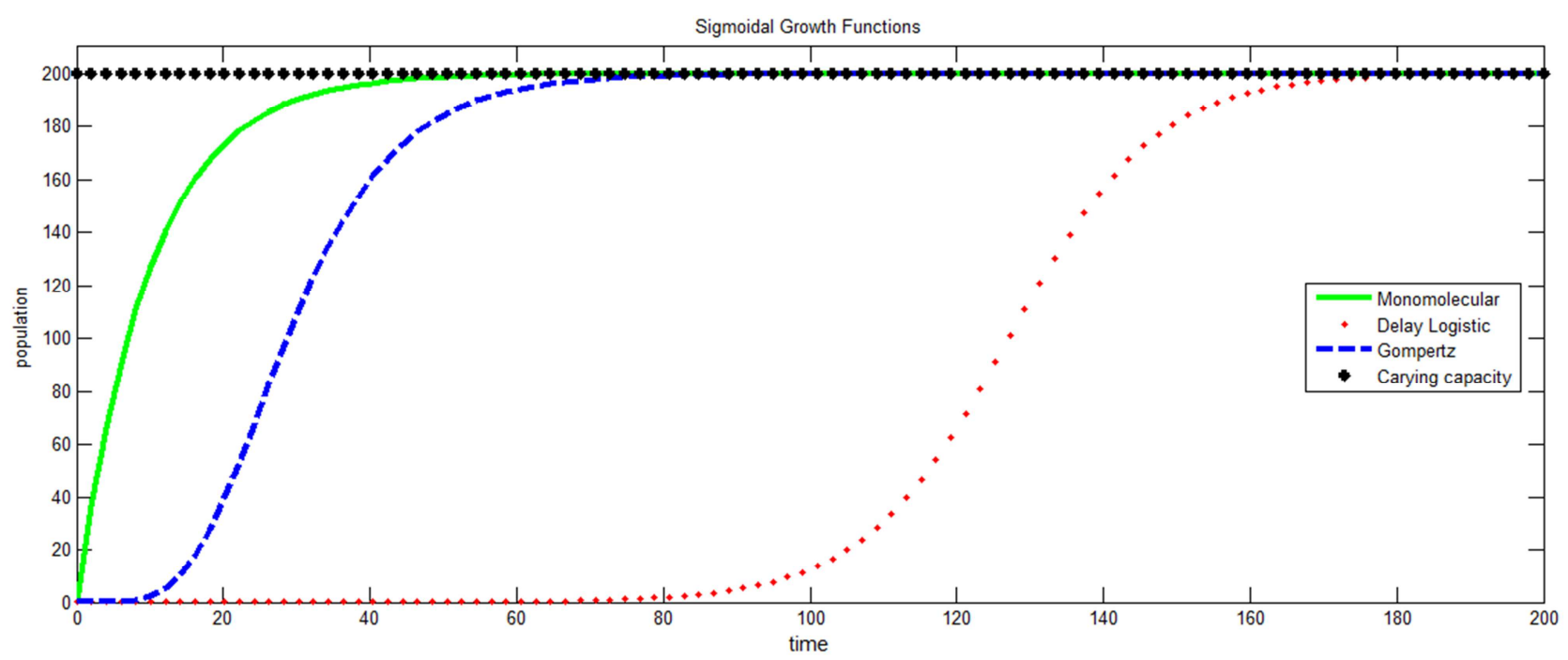

Figure 2. Sigmoid Growth of functions.

\subsection{Application of Monomolecular Function}

Monomolecular growth function $f(t)=K\left(1-\mathrm{C} e^{-r t}\right)$ is one of Koya-Goshu general biological growth function that gives a growth rate of $\frac{d f(t)}{d t}=r(K-f(t))$. Monomolecular function is a well-known model of biological population with saturation of their ecosystem like tumor growth. The monomolecular growth function is applicable on the heterogeneous model of avascular tumor growth proposed (1) for this work. Here the function has meaningful impact on the proliferating cells of the tumor in its spheroid that change first entity of the model. Now the model on dynamics of avascular tumor growth with the application of monomolecular function provides a rate change on its mitosis so that its first equation becomes

$$
\frac{d P}{\mathrm{dt}}=\left[\mathrm{R}\left(\frac{K}{\mathrm{P}}-1\right)-\mathrm{R}_{1}-\mu_{\mathrm{p}}\right] \mathrm{P}+\mathrm{R}_{2} \mathrm{Q}
$$

\subsection{Application of Logistic Delay Function}

A general class of models used for quantification of tumor growth kinetics has a sigmoid shape which is an increasing curve with one inflection point that asymptotically converges to maximal value, is basically logistic model [11]. The logistic growth function $f(t)=\frac{K}{1+\mathrm{C} e^{-r t}}$ which yields a rate of $\frac{d f(t)}{d t}=r f(t)\left(1-\frac{f(t-\tau)}{K}\right)$ at a delay time $\tau$ earlier is a good model of biological population growth in species which have grown relatively too large that they are near to saturating their ecosystem like tumor growth. This delay logistic expression is applicable on the model of avascular tumor growth proposed and as a result of altering the proliferating cells of the spheroid, the heterogeneous model of avascular tumor growth can be restated simply by setting ODE as

$$
\frac{d P}{\mathrm{dt}}=\left[\mathrm{R}\left(1-\frac{\mathrm{P}(\mathrm{t}-\tau)}{\mathrm{K}}\right)-\mathrm{R}_{1}-\mu_{\mathrm{p}}\right] \mathrm{P}+\mathrm{R}_{2} \mathrm{Q}
$$

\subsection{Application of Gompertz Function}

The Gompertz function describes global dynamics of many natural processes including growth of normal and malignant tissue [10]. The Gompertz model became an accepted representation of growth processes in general and of 
tumor growth in particular. The essential characteristic of the Gompertz model is that it exhibits exponential decay of relative growth rate. Dynamics of growth of normal or malignant cells is in general described by the Gompertz function (Winsor, 1932) defined as $f(t)=K e^{C \exp (-r t)}$ with a rate of $\frac{d f(t)}{d t}=r f(t) \log \frac{K}{f(t)}$ which is applied in the heterogeneous model on dynamics of avascular tumor growth so as to modify the proposed model leads to the following change for (1a).

$$
\frac{d Q}{\mathrm{dt}}=\left[\mathrm{R} \log \frac{K}{\mathrm{P}}-\mathrm{R}_{1}-\mu_{\mathrm{p}}\right] \mathrm{P}+\mathrm{R}_{2}
$$

\section{Stability Analysis}

In this section we analyze the general heterogonous model of avascular tumor growth together with the modified systems as the application of growth function using Jacobian matrix at their equilibrium point by Routh-Hurwith eigenvalues test evaluation.

\subsection{General Heterogonous Model}

\subsubsection{Equilibrium Points of the Model}

Considering the heterogonous model (1) of this work, it is not tedious to find the Free State equilibrium point is $E_{0}=$ $(0,0,0)$. Moreover, the nontrivial equilibrium point derived from the relation $\frac{d P}{\mathrm{dt}}=\frac{d Q}{\mathrm{dt}}=\frac{d D}{\mathrm{dt}}=0$ can be simply represented by $E^{*}=\left(P^{*}, Q^{*}, D^{*}\right)$.

\subsubsection{Stability Analysis of the Model}

Since a plenty of nutrient is available at the onset of tumor growth, tumor cells are expected to proliferate freely with no need to transform into the quiescent stage [4] and the exiting cells are not considerably counted. Hence we assume $\mathrm{R}_{1}(0)=0$. Based on this assumption and by considering the free equilibrium point, the local stability of $E_{0}=(0,0,0)$ can be determined by system of Jacobian matrix.

$$
\begin{gathered}
J\left(E_{0}\right)=\left(\begin{array}{ccc}
{\left[R-\mathrm{R}_{1}-\mu_{\mathrm{p}}\right]} & \mathrm{R}_{2} & 0 \\
\mathrm{R}_{1} & -\left[\mathrm{R}_{2}+\mathrm{R}_{3}+\mu_{\mathrm{q}}\right] & 0 \\
0 & \mathrm{R}_{3} & -\mu_{\mathrm{d}}
\end{array}\right) \\
=\left(\begin{array}{ccc}
R & \mathrm{R}_{2} & 0 \\
0 & -\left[\mathrm{R}_{2}+\mathrm{R}_{3}\right] & 0 \\
0 & \mathrm{R}_{3} & -\mu_{\mathrm{d}}
\end{array}\right) \\
(\mathrm{R}-\lambda)\left(-\mathrm{R}_{2}-\mathrm{R}_{3}-\lambda\right)\left(-\mu_{\mathrm{d}}-\lambda\right)=0 \\
\lambda_{1}=R>0, \quad \lambda_{2}=-\left[\mathrm{R}_{2}+\mathrm{R}_{3}\right], \quad \lambda_{3}=-\mu_{\mathrm{d}}
\end{gathered}
$$

Based on the Routh-Hurwith perspective, as all the eigenvalues of the Jacobian matrix have no negative real parts, the model at the free equilibrium point is saddle point which is not stable. Indeed the other derived models as the application of the biological growth functions are not also stable at the trivial state. On the other hand the nontrivial steady state of the model at $E^{*}=\left(P^{*}, Q^{*}, D^{*}\right)$ is determined by the Jacobian matrix too.

$$
J\left(E^{*}\right)=\left(\begin{array}{ccc}
{\left[R-\mathrm{R}_{1}-\mu_{\mathrm{p}}\right]} & \mathrm{R}_{2} & 0 \\
\mathrm{R}_{1} & -\left[\mathrm{R}_{2}+\mathrm{R}_{3}+\mu_{\mathrm{q}}\right] & 0 \\
0 & \mathrm{R}_{3} & -\mu_{\mathrm{d}}
\end{array}\right)
$$

Now let $x=R-\mathrm{R}_{1}-\mu_{\mathrm{p}}$, and $y=-\left[\mathrm{R}_{2}+\mathrm{R}_{3}+\mu_{\mathrm{q}}\right]$. At the equilibrium point of the model the Jacobian matrix becomes:

$$
J\left(P^{*}, Q^{*}, D^{*}\right)=\left(\begin{array}{ccc}
x & \mathrm{R}_{2} & 0 \\
\mathrm{R}_{1} & y & 0 \\
0 & \mathrm{R}_{3} & -\mu_{\mathrm{d}}
\end{array}\right)
$$

Using technique of determinant we can determine the eigenvalues of the system and they are:

$$
\begin{aligned}
\lambda_{1}=-\mu_{\mathrm{d}}<0, \quad & \lambda_{2}=\frac{(x+y)-\sqrt{(x+y)^{2}+4 \mathrm{R}_{1} \mathrm{R}_{2}}}{2} \\
= & \frac{(x+y)-(x+y) \sqrt{1+\frac{4 \mathrm{R}_{1} \mathrm{R}_{2}}{(x+y)^{2}}}}{2}<0
\end{aligned}
$$

$$
\begin{aligned}
\lambda_{3}=\frac{(x+y)+\sqrt{(x+y)^{2}+4 \mathrm{R}_{1} \mathrm{R}_{2}}}{2} \\
=\frac{(x+y)+(x+y) \sqrt{1+\frac{4 \mathrm{R}_{1} \mathrm{R}_{2}}{(x+y)^{2}}}}{2} .
\end{aligned}
$$

Based on $\lambda_{3}$, Routh-Hurwith holds at:

$$
x+y=R-\left[\mathrm{R}_{1}+\mu_{\mathrm{p}}+\mathrm{R}_{2}+\mathrm{R}_{3}+\mu_{\mathrm{q}}\right]<0 .
$$

Considering maximum proliferating rate $\mathrm{R}=0.04$ /day, apoptosis rate remains constant 0.02 per day at each state [13], we compute parameter values based on functional relations expressed by $c=\frac{c_{0} \gamma}{\gamma+P}(1+\alpha(P+Q+D)), \mathrm{R}_{1}=$ $\frac{1}{2}\left(1-\tanh (4 c-2), \mathrm{R}_{3}=\frac{1}{2}\left(\mathrm{R}_{1}\right), \mathrm{R}=\beta e^{\beta \mathrm{c}}[7,12]\right.$ provide $\mathrm{R}_{1}=1, \mathrm{R}_{2}=0.48, \mathrm{R}_{3}=0.5$ at which the steady state of the systems are evaluated. Hence, the value of $x+y$ is $-1.98<0$ that indicates the model is stable. Biologically the model is more suitable when the rates of quiescence and necrosis are not less than or simply exceeds the mitosis and the recurred quiescent cells for which nutrient reach and hence we can conclude the model fits with condition of tumor.

\subsection{Monomolecular Function}

\subsubsection{Equilibrium Point of Monomolecular Model}

To find out the nontrivial equilibrium point $E^{*}=$ $\left(P^{*}, Q^{*}, D^{*}\right)$ we start from equation (1c) then we get $Q^{*}=$ $\frac{\mu_{\mathrm{d}}}{\mathrm{R}_{3}} D^{*}$ and from equation (1b) we have $P^{*}=\left[\mathrm{R}_{2}+\mathrm{R}_{3}+\right.$ $\left.\mu_{\mathrm{q}}\right] \frac{\mu_{\mathrm{d}}}{\mathrm{R}_{1} \mathrm{R}_{3}} D^{*}$. Using these relations with equation (1a) based on the application of monomolecular growth function we determine $P^{*}, Q^{*}$ and $D^{*}$ from $\mathrm{RK}-\left[\mathrm{R}+\mu_{\mathrm{p}}+\mathrm{R}_{1}\right] P^{*}+$ $\mathrm{R}_{2} Q^{*}=0$ as follow:

$$
\begin{aligned}
& D^{*}=\frac{\mathrm{RKR}_{1} \mathrm{R}_{3}}{\left[\left(\mathrm{R}+\mu_{\mathrm{p}}+\mathrm{R}_{1}\right)\left(\mathrm{R}_{2}+\mathrm{R}_{3}+\mu_{\mathrm{q}}\right)-\mathrm{R}_{1} \mathrm{R}_{2}\right] \mu_{\mathrm{d}}}, \\
& Q^{*}=\frac{\mathrm{RKR}_{1}}{\left(\mathrm{R}+\mu_{\mathrm{p}}+\mathrm{R}_{1}\right)\left(\mathrm{R}_{2}+\mathrm{R}_{3}+\mu_{\mathrm{q}}\right)-\mathrm{R}_{1} \mathrm{R}_{2}}, \text { and }
\end{aligned}
$$




$$
P^{*}=\frac{\mathrm{RK}\left(\mathrm{R}_{2}+\mathrm{R}_{3}+\mu_{\mathrm{q}}\right)}{\left(\mathrm{R}+\mu_{\mathrm{p}}+\mathrm{R}_{1}\right)\left(\mathrm{R}_{2}+\mathrm{R}_{3}+\mu_{\mathrm{q}}\right)-\mathrm{R}_{1} \mathrm{R}_{2}}
$$$$
\text { Now let } \beta=\frac{\mathrm{RK}}{\left[\left(\mathrm{R}+\mu_{\mathrm{p}}+\mathrm{R}_{1}\right)\left(\mathrm{R}_{2}+\mathrm{R}_{3}+\mu_{\mathrm{q}}\right)-\mathrm{R}_{1} \mathrm{R}_{2}\right] \mu_{\mathrm{d}}} \text {. }
$$

Thus the equilibrium point is given by

$$
\left(P^{*}, Q^{*}, D^{*}\right)=\left(\beta \mu_{\mathrm{d}}\left[\mathrm{R}_{2}+\mathrm{R}_{3}+\mu_{\mathrm{q}}\right], \beta \mathrm{R}_{1}, \beta \mathrm{R}_{1} \mathrm{R}_{3}\right)
$$

\subsubsection{Stability Analysis of Monomolecular Model}

Based on the given functions of the model that matrix at any point including at $E^{*}$ will be

$$
\begin{gathered}
J(P, Q, D)=J\left(P^{*}, Q^{*}, D^{*}\right) \\
=\left(\begin{array}{ccc}
-\left[\mathrm{R}+\mu_{\mathrm{p}}+\mathrm{R}_{1}\right] & \mathrm{R}_{2} & 0 \\
\mathrm{R}_{1} & -\left[\mathrm{R}_{2}+\mathrm{R}_{3}+\mu_{\mathrm{q}}\right] & 0 \\
0 & \mathrm{R}_{3} & -\mu_{\mathrm{d}}
\end{array}\right)
\end{gathered}
$$

Now let $x=-\left(\mathrm{R}+\mu_{\mathrm{p}}+\mathrm{R}_{1}\right)$, and $y=-\left[\mathrm{R}_{2}+\mathrm{R}_{3}+\right.$ $\mu_{\mathrm{q}}$ ] such that the eigenvalues becomes:

$$
\begin{gathered}
\lambda_{1}=-\mu_{\mathrm{d}}<0, \quad \lambda_{2}=\frac{(x+y)-(x+y) \sqrt{1+\frac{4 \mathrm{R}_{1} \mathrm{R}_{2}}{(x+y)^{2}}}<0, \text { and }}{2} \\
\lambda_{3}=\frac{(x+y)+(x+y) \sqrt{1+\frac{4 \mathrm{R}_{1} \mathrm{R}_{2}}{(x+y)^{2}}}}{2}
\end{gathered}
$$

Considering the third eigenvalues $\lambda_{3}$, clearly $x+y<0$ implies that $-\left[R_{2}+R+\mu_{p}+R_{1}+R_{3}+\mu_{q}\right]$ is negative and the model is stable.

\subsection{Delay Logistic Function}

\subsubsection{Equilibrium Point of Time Delay Logistic Model}

Like in a monomolecular function the nontrivial equilibrium point $E^{*}=\left(P^{*}, Q^{*}, D^{*}\right)$ of the time delay logistic we get relations $Q^{*}=\frac{\mu_{\mathrm{d}}}{\mathrm{R}_{3}} D^{*}$ and $P^{*}=\left[\mathrm{R}_{2}+\mathrm{R}_{3}+\right.$ $\left.\mu_{\mathrm{q}}\right] \frac{\mu_{\mathrm{d}}}{\mathrm{R}_{1} \mathrm{R}_{3}} D^{*}$. Now let $\delta=\frac{\mathrm{R}_{2}+\mathrm{R}_{3}+\mu_{\mathrm{q}}}{\mathrm{R}_{1} \mathrm{R}_{3}}$, so that the value of $P^{*}$ will reduce to $P^{*}=\delta \mu_{\mathrm{d}} D^{*}$. According to these relations and delay logistic proliferating component of the model for a small time delay, we have $\frac{\mathrm{R}}{\mathrm{K}} P^{* 2}+\left(\mu_{\mathrm{p}}+\mathrm{R}_{1}-\mathrm{R}\right) P^{*}-$ $\mathrm{R}_{2} Q^{*}=0$ and its equilibrium point yields:

$$
\begin{gathered}
D^{*}=\mathrm{K} \frac{\delta\left(\mathrm{R}-\mathrm{R}_{1}-\mu_{\mathrm{p}}\right)+\frac{\mathrm{R}_{2}}{\mathrm{R}_{3}}}{\delta^{2} \mathrm{R} \mu_{\mathrm{d}}}, Q^{*}=\mathrm{K} \frac{\delta\left(\mathrm{R}-\mathrm{R}_{1}-\mu_{\mathrm{p}}\right)+\frac{\mathrm{R}_{2}}{\mathrm{R}_{3}}}{\delta^{2} \mathrm{RR}_{3}} \text {, and } P^{*}= \\
\mathrm{K} \frac{\delta\left(\mathrm{R}-\mathrm{R}_{1}-\mu_{\mathrm{p}}\right)+\frac{\mathrm{R}_{2}}{\mathrm{R}_{3}}}{\delta \mathrm{R}} .
\end{gathered}
$$

Suppose $\mathcal{W}=\frac{\delta\left(\mathrm{R}-\mathrm{R}_{1}-\mu_{\mathrm{p}}\right)+\frac{\mathrm{R}_{2}}{\mathrm{R}_{3}}}{\delta}$, the equilibrium points of the tumor growth model with application of delay function are given by $\left(P^{*}, Q^{*}, D^{*}\right)=\left(\frac{\mathrm{K} \mathcal{W}}{\mathrm{R}}, \frac{\mathrm{K} \mathcal{W}}{\delta \mathrm{RR}_{3}}, \frac{\mathrm{K} \mathcal{W}}{\delta \mathrm{R} \mu_{\mathrm{d}}}\right)$

\subsubsection{Stability Analysis of Time Delay Logistic Model}

Based on the given functions of the model the matrix is:
$J(P, Q, D)$

$=\left(\begin{array}{ccc}{\left[\frac{-2 \mathrm{R}}{\mathrm{K}} P+\mathrm{R}-\mu_{\mathrm{p}}-\mathrm{R}_{1}\right]} & \mathrm{R}_{2} & 0 \\ \mathrm{R}_{1} & -\left[\mathrm{R}_{2}+\mathrm{R}_{3}+\mu_{\mathrm{q}}\right] & 0 \\ 0 & \mathrm{R}_{3} & -\mu_{\mathrm{d}}\end{array}\right)$

At the equilibrium point of the model the Jacobian matrix becomes

$$
\begin{gathered}
J\left(P^{*}, Q^{*}, D^{*}\right) \\
=\left(\begin{array}{ccc}
{\left[-2 \mathcal{W}+\mathrm{R}-\mu_{\mathrm{p}}-\mathrm{R}_{1}\right]} & \mathrm{R}_{2} & 0 \\
\mathrm{R}_{1} & -\left[\mathrm{R}_{2}+\mathrm{R}_{3}+\mu_{\mathrm{q}}\right] & 0 \\
0 & \mathrm{R}_{3} & -\mu_{\mathrm{d}}
\end{array}\right)
\end{gathered}
$$

For $x=-2 \mathcal{W}+\mathrm{R}-\mu_{\mathrm{p}}-\mathrm{R}_{1}$, and $y=\mathrm{R}_{2}-\mathrm{R}_{3}-\mu_{\mathrm{q}}$, the eigenvalues of the matrix are:

$$
\begin{gathered}
\lambda_{1}=-\mu_{\mathrm{d}}<0, \quad \lambda_{2}=\frac{(x+y)-(x+y) \sqrt{1+\frac{4 \mathrm{R}_{1} \mathrm{R}_{2}}{(x+y)^{2}}}}{2}<0 \text { and } \\
\lambda_{3}=\frac{(x+y)+(x+y) \sqrt{1+\frac{4 \mathrm{R}_{1} \mathrm{R}_{2}}{(x+y)^{2}}}}{2} .
\end{gathered}
$$

From $\lambda_{3}$, the model is stable at $x+y<0$.

Here $x+y=-1.48<0$ based on the rates used in the above systems (1 a-c).

\subsection{Gompertz Function}

\subsubsection{Equilibrium Point of Gompertz Model}

Clearly $Q^{*}=\frac{\mu_{\mathrm{d}}}{\mathrm{R}_{3}} D^{*}$ and $P^{*}=\left[\mathrm{R}_{2}+\mathrm{R}_{3}+\mu_{\mathrm{q}}\right] \frac{\mu_{\mathrm{d}}}{\mathrm{R}_{1} \mathrm{R}_{3}} D^{*}$ for $\delta=\frac{\mathrm{R}_{2}+\mathrm{R}_{3}+\mu_{\mathrm{q}}}{\mathrm{R}_{1} \mathrm{R}_{3}}$. Based on the relations above as well as the equation (1a) and $\frac{d P}{d t}=0$ the proliferating component of its equilibrium point gives $\mathrm{P}^{*}=K \boldsymbol{e}^{\frac{1}{\mathrm{R}}\left(\frac{\mathrm{R}_{2}}{\delta \mathrm{R}_{3}}-\mu_{\mathrm{p}}-\mathrm{R}_{1}\right)}$. So that $\mathrm{D}^{*}=\frac{P^{*}}{\delta \mu_{\mathrm{d}}}=\frac{K}{\delta \mu_{\mathrm{d}}} \boldsymbol{e}^{\frac{1}{\mathrm{R}}\left(\frac{\mathrm{R}_{2}}{\delta \mathrm{R}_{3}}-\mu_{\mathrm{p}}-\mathrm{R}_{1}\right)}, \quad$ and $\quad \mathrm{Q}^{*}=\frac{\mu_{\mathrm{d}}}{\mathrm{R}_{3}} \mathrm{D}^{*}=$ $\frac{K}{\delta \mathrm{R}_{3}} e^{\frac{1}{\mathrm{R}}\left(\frac{\mathrm{R}_{2}}{\delta \mathrm{R}_{3}}-\mu_{\mathrm{p}}-\mathrm{R}_{1}\right)}$.Let $\phi=\frac{\mathrm{R}_{2}}{\delta \mathrm{R}_{3}}-\mu_{\mathrm{p}}-\mathrm{R}_{1}$, the equilibrium point is $\left(P^{*}, Q^{*}, D^{*}\right)=\left(K \boldsymbol{e}^{\frac{\phi}{\mathrm{R}}}, \frac{K}{\delta \mathrm{R}_{3}} \boldsymbol{e}^{\frac{\phi}{\mathrm{R}}}, \frac{K}{\delta \mu_{\mathrm{d}}} \boldsymbol{e}^{\frac{\phi}{\mathrm{R}}}\right)$.

\subsubsection{Stability Analysis of Gompertz Model}

The partial derivative of $f$ with respect to $\mathrm{P}$ is given by $\frac{\partial f}{\partial P}=\mathrm{R} \log \frac{K}{\mathrm{P}}-\mathrm{R}-\mu_{\mathrm{p}}-\mathrm{R}_{1}$. Based on the given functions of the model the Jacobian matrix will be

$$
\begin{aligned}
& J(P, Q, D) \\
& =\left(\begin{array}{ccc}
{\left[\mathrm{R} \log \frac{K}{\mathrm{P}}-\mathrm{R}-\mu_{\mathrm{p}}-\mathrm{R}_{1}\right]} & \mathrm{R}_{2} & 0 \\
\mathrm{R}_{1} & -\left[\mathrm{R}_{2}+\mathrm{R}_{3}+\mu_{\mathrm{q}}\right] & 0 \\
0 & \mathrm{R}_{3} & -\mu_{\mathrm{d}}
\end{array}\right)
\end{aligned}
$$

At $E^{*}$, proliferating value becomes: $P^{*}=\mathrm{R} \log \frac{K}{\mathrm{~K}} \boldsymbol{e}^{\left(\frac{-\phi}{\mathrm{R}}\right)}-\mathrm{R}-\mu_{\mathrm{p}}-\mathrm{R}_{1}=-\phi-\mathrm{R}-\mu_{\mathrm{p}}-\mathrm{R}_{1}$. So that the Jacobian matrix at the equilibrium point is: 
$J\left(P^{*}, Q^{*}, D^{*}\right)=\left(\begin{array}{ccc}-\left[\phi+\mathrm{R}+\mu_{\mathrm{p}}+\mathrm{R}_{1}\right] & \mathrm{R}_{2} & 0 \\ \mathrm{R}_{1} & -\left[\mathrm{R}_{2}+\mathrm{R}_{3}+\mu_{\mathrm{q}}\right] & 0 \\ 0 & \mathrm{R}_{3} & -\mu_{\mathrm{d}}\end{array}\right)$

For $x=-\left[\frac{\mathrm{R}_{2}}{\delta \mathrm{R}_{3}}-\mu_{\mathrm{p}}-\mathrm{R}_{1}\right]-R-\mu_{\mathrm{p}}-\mathrm{R}_{1}$, and $y=-\left[\mathrm{R}_{2}+\mathrm{R}_{3}+\mu_{\mathrm{q}}\right]$, all the three eigenvalues are negative if $x+y<0$,and the parameter values above match with this situation, $x+y=-1.52<0$ so that stability of the model exists.

\section{Simulation Study}

\subsection{Growth Functions Calibration}

In this section, we compare the three biological growth functions stated above by observing their graphical results with an experimental data. In this discussion, the data chosen are the experimental results from [14] manipulated in a mouse used to compare the growth functions for carrying capacity $\mathrm{K}=150 \times 10^{7}$ and rate of growth $\mathrm{R}=0.6$ perday. Graphs of the data and results obtained from the calibrated growth functions are shown in Figure 3.

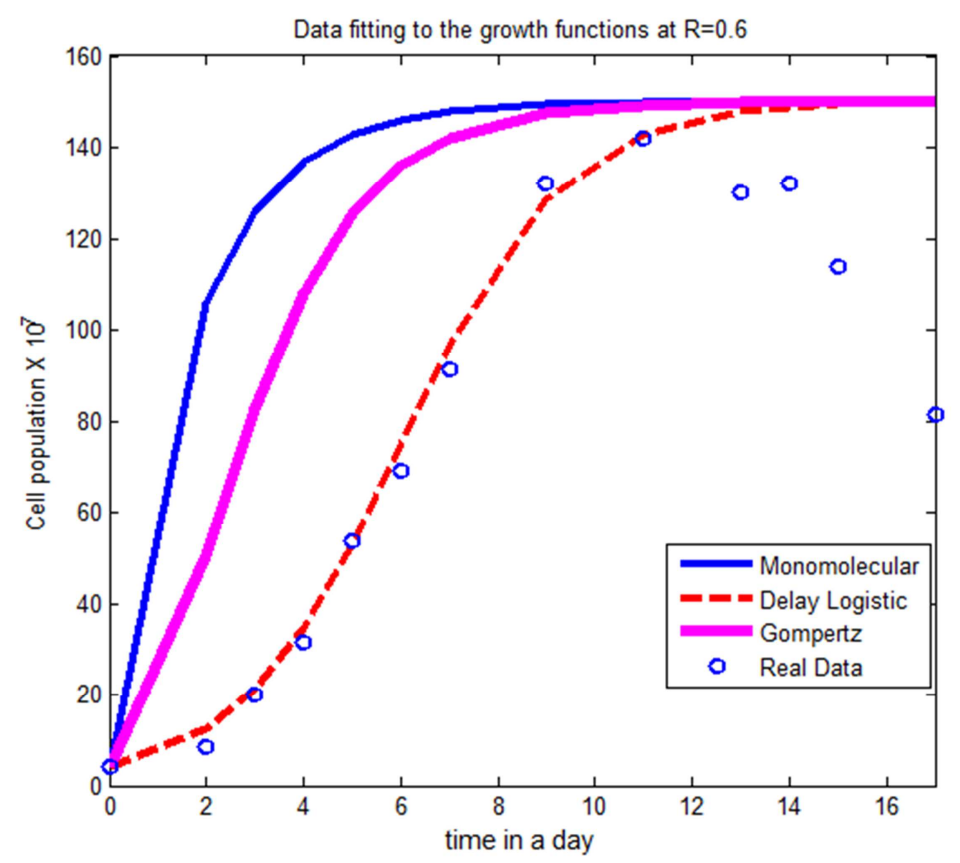

Figure 3. Growth functions calibration at $R=0.6$.

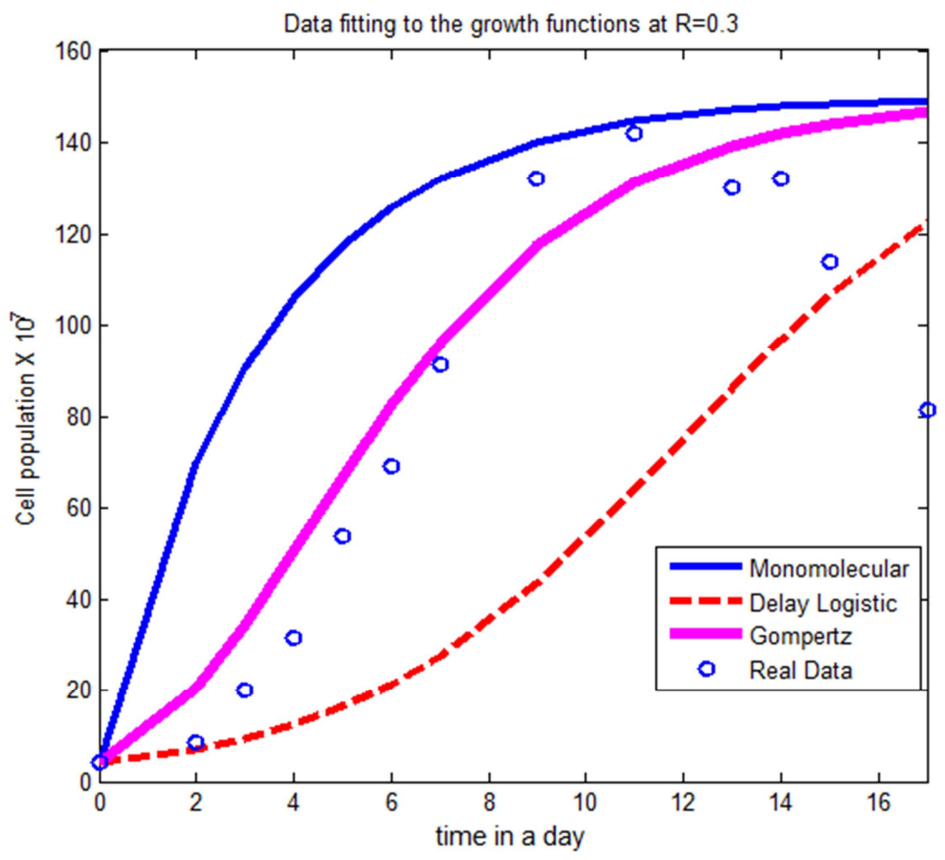

Figure 4. Growth functions calibration at $R=0.3$. 
The results of growth functions compare quite well with the experimental data and they all have similar path with the direction of data growth. The time delay logistic growth function fits more closely with the data as in figure 3. Gompertz is the next and monomolecular is the least to fit with this particular data. However, this rate of proliferating is very high that may be when there are enough nutrients around the tumor. With the presence of nutrient limitation the rate becomes too less. For example, if the rate is reduced say to half $(\mathrm{R}=0.3)$, the data mostly fits with Gompertz as we show in figure 4.

\subsection{Model Simulation}

We notice the following phenomena of the three biological growth functions discussed above, which clearly shows avascular tumor growth. The functional forms and parameter values are chosen from earlier studies to validate the models stated in table 3 below.

Table 3. Functional forms parameter values of the tumor growth model.

\begin{tabular}{llll}
\hline Functional forms of parameters & Reference & Parameter values & Reference \\
\hline $\mathrm{R}=\mathrm{R}(\mathrm{c})=\beta e^{\beta c}$ & {$[7,12]$} & $c_{0}=1$ & {$[7,12]$} \\
$\mathrm{R}_{1}=\mathrm{R}_{1}(c)=\frac{1}{2}(1-\tanh (4 c-2))$ & {$[7,12]$} & $\alpha=0.2, \beta=0.8, \gamma=10$ & {$[7,12]$} \\
$\mathrm{R}_{3}=\mathrm{R}_{3}(c)=\frac{1}{2}\left(\mathrm{R}_{1}\right)$ & {$[7,12]$} & $\mathrm{K}=10^{6}$ & {$[12]$} \\
$c=\frac{c_{0} \gamma}{\gamma+P}(1+\alpha(P+Q+D))$ & {$[7,12]$} & $\mu_{\mathrm{p}}=\mu_{\mathrm{q}}=\mu_{\mathrm{d}}=0.02$ & {$[13]$} \\
$\mathrm{R}_{2}=\mathrm{R}_{2}(c)=\frac{1}{2}\left(\mathrm{R}_{1}\right)-\mu_{\mathrm{p}}$ & Computed & $\tau=0.009$ & Assumption \\
\hline
\end{tabular}

Our results confirm previous observations [1-14] that tumor growth is not a simple exponential function. The biological growth functions like monomolecular, delay logistic, and Gompertz functions give a better description of growth slowdown for the status of tumor growth. From figure 5 we observe that in all cases proliferating subpopulation is dominant initially, but when time passes as a consequence of nutrient insufficiency the concentration of quiescent and necrotic cells exceed the reproductive proliferating cells as a result of their mutation. This is biologically justified to describe tumor growth as a cause of different cancer diseases.
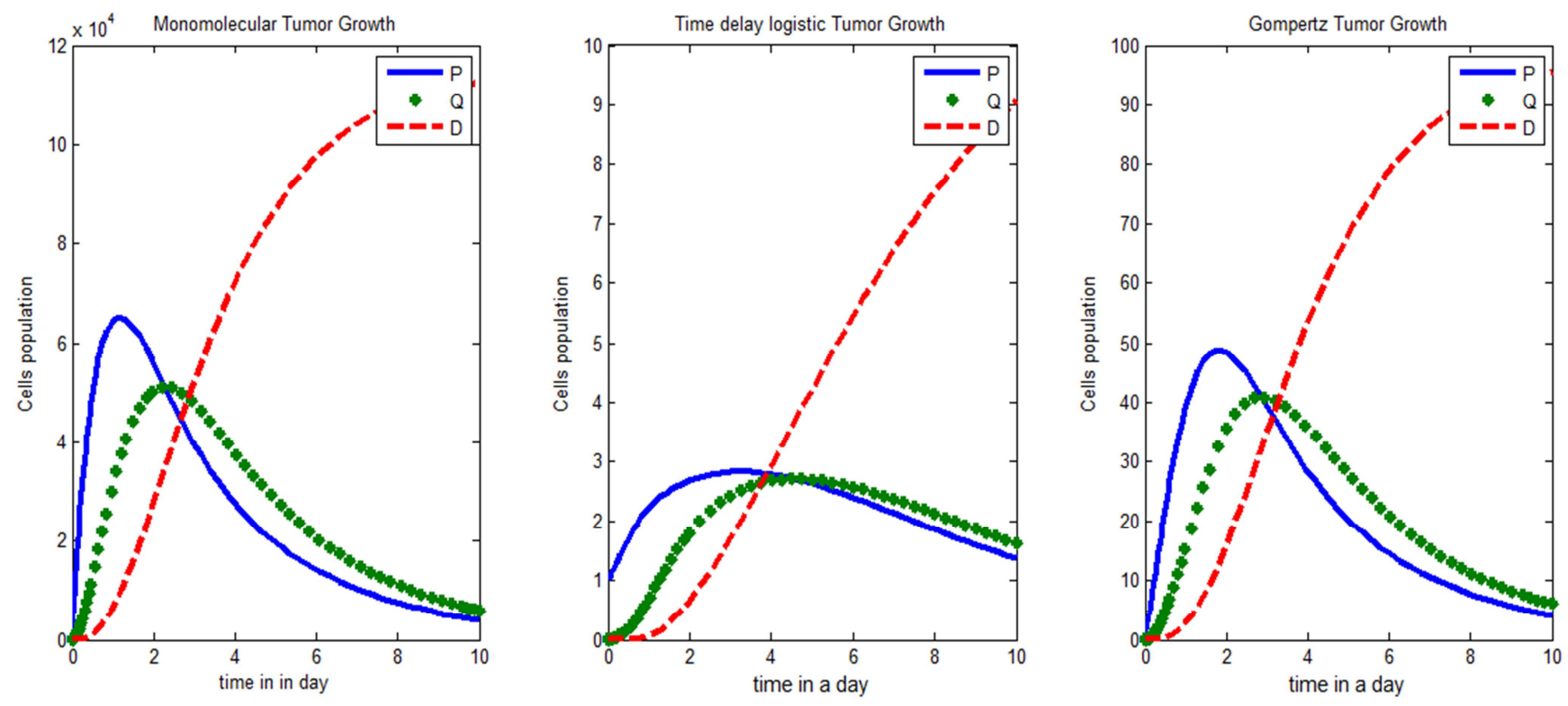

Figure 5. Tumor Growth with proliferating, quiescent and necrotic components.

Despite the structural similarities of the three systems, there are important differences in their simulation outputs. In the system of the model with monomolecular function, there is high proliferating rate that leads to high rates of quiescence and necrosis too. However, the model with time delay logistic has slow rate and the Gompertz base model shows an average rate.

\section{Conclusions}

As stated in the introduction mathematical modeling and analysis of tumor growth processes give important insights on the most worldwide dangerous cancerdisease. Considering the three components of a tumor, the proliferating, quiescent and necrotic cells explicitly in its spheroidand our work deals 
on a three compartmental model. We then applied three growth functions known as monomolecular, time delay logistic, and Gompertz which influence on mitosis parametric rate that affects the proliferating parts of the spheroid. Because of the fact that tumor cells are limited by resources mainly the nutrient supply, we use nutrient based parameters.

Wehave analyzed the heterogonous model of avascular tumor growth together with the modified systems as the application of growth function using Jacobian matrix at their equilibrium by the Routh-Hurwith eigenvalues test. We find that the model at the free equilibrium point is saddle which is not stable, whereas the nontrivial steady state of the model is stable whatever any growth function is applied.

The simulationsare done also in general have the proliferating growth of cells and as a result of spheroid enlargement which leads to the nutrient insufficiency resulting cells to mutate in to quiescent and then to necrotic stage. In early stages of the growth, the proliferating rate is a large fraction of the spheroid, however, as time passes the growth rate of proliferating cells is reduced drastically, whereas the numbers of dead cells maximize to be accumulated at the core of the spheroid that might be malignant tumor.

\section{Acknowledgments}

We wish to express our gratitude and thanks to School of Mathematical and Statistical sciences, Hawassa University for providing this opportunity to complete the work.

\section{References}

[1] Anna Kane Laird, "Dynamics of tumor growth", Biological and medical research, Argonne National Laboratory, USA, (June 8, 1964).

[2] Anne Talkington and Rick Durrett, "Estimating tumor growth rates in vivo" Dept. of Math, Duke University, Durham, NC (November 19, 2014) 1-27.

[3] Arseniy S. Karkach, "Trajectories and models of individual", Demographic Research VOLUME 15, ARTICLE 12, PAGES 347-400 (7 NOV. 2006).

[4] E. O. Alzahrani, AsimAsiri, M. M. El-Dessoky, Y. Kuang, "Quiescence as an explanation of Gompertzian tumor growth revisited", Mathematical Biosciences 254 (2014) 76-82.

[5] Frank Kozusko, Zeljko Bajzer, "Combining Gompertzian growth and cell population dynamics", www.elsevier.com/locate/mbs, Mathematical Biosciences 185 (2003) 153-167.
[6] Heiko Enderling and Mark A. J Chaplain, "Mathematical Modeling of Tumor Growth and Treatment", USA, (2014).

[7] Keng-Cheng Ang, "Analysis of tumor growth with MATLAB", National Institute of education, Nanyang Technological University, Singapore.

[8] Nicholas F. Britton, "Essential Mathematical Biology", Department of Mathematical Sciences, University of Bath, Claverton Down, Bath BA2 7AY, UK, (2003).

[9] Purnachandra Rao Koya, Ayele Taye Goshu, "Generalized Mathematical Model for Biological Growth", Open Journal of Modeling and Simulation, 2013, 1, 4253.http://dx.doi.org/10.4236/ojmsi.2013.14008.

[10] P. Waliszewski, J. Konarski, "A Mystery of the Gompertz Function" Dept. of Theoretical Chemistry, University of Poznan, Grunwaldzka 6, 60-780 Poznan, Poland, 277-286.

[11] Sebastein Benzekry, Clare Lamont, Afshin Beheshti, Amanda Tracz, John M. L. Ebos, Lynn Hlatky, Philip Hahnfeldt," Classical Mathematical Models for Descriptionand Prediction of Experimental Tumor Growth", sebastien.benzekry@inria.fr, $1-56$.

[12] Tan Liang Soon and AngKeng Cheng, "A numerical simulation of avascular tumor growth", Nanyang Tech. University, Singapore, ANZIAM J.46 (E) pp. C909-C917, (2005).

[13] Travis Portz, Yang Kuang, and John D. Nagy, “A clinical data validated mathematical model of prostate cancer growth under intermittent androgen suppression therapy" AIP ADVANCES 2, 011002 (2012).

[14] Urszula Forys, Anna Marcinisk-Czochra, "Logistic EquationsIn Tumor Growth Modeling" Int. J. Appl. Math. Comp. Sci., 2003, Vol. 13, No. 3, 317-325.

[15] Purnachandra Rao Koya, Ayele Taye Goshu and Mohammed Yiha Dawed. Modelling Predator Population assuming that the prey follows Richards Growth model. European Journal of Academic Essays (EJAE). Vol. 1, No. 9, 2014, pp. 42-51. http://euroessays.org/wp-content/uploads/2014/10/EJAE279.pdfhttp://euroessays.org/archieve/vol-1-issue-9.

[16] Ayele Taye Goshu, Purnachandra Rao Koya. Predator Population Dynamics Involving Exponential Integral Function when Prey follows Gompertz Model. Open Journal of Modelling and Simulation (OJMSi). Vol. 3, 2015, pp 70-80. http://dx.doi.org/10.4236/ojmsi.2015.33008.

[17] Mohammed Yiha Dawed, Purnachandra Rao Koya, Temesgen Tibebu. Analysis of prey - predator system with prey population experiencing critical depensation growth function. American Journal of applied Mathematics. Vol. 3, No. 6, 2015, Pp. 327-334. Doi: 10.11648/j.ajam.20150306.23. 